\title{
Preface: Special Issue of Selected Extended Papers of CADE-22
}

\author{
Renate A. Schmidt • Brigitte Pientka
}

Received: 30 November 2010 / Accepted: 1 December 2010 / Published online: 22 December 2010

(C) Springer Science+Business Media B.V. 2010

This special issue of the Journal of Automated Reasoning is dedicated to a selection of papers presented at The 22nd International Conference on Automated Deduction (CADE-22), held at McGill University in Montreal, Canada, in August 2009. CADE is the major forum for the presentation of research in all aspects of automated deduction, from foundations and applications to implementations and practical experiences.

The papers selected for this special issue underwent a two-round reviewing process. In the first round, the CADE-22 programme committee selected 32 technical papers and system descriptions out of 77 initial submissions for publication in the proceedings of the conference [1]. From the best papers in the proceedings as evaluated by the reviewers we invited the authors of seven papers to submit revised and extended version of their papers to this special issue. In the second round, the submitted extended papers were reviewed using the normal reviewing process for journals in accordance with Journal of Automated Reasoning requirements. Each paper was reviewed by either three or four reviewers, many of whom were new reviewers.

The four selected papers in this special issue concentrate on traditional core areas at the forefront of automated deduction. It is coincidental, but not unsurprising, that all have in common that they combine techniques and tools from different logical settings and different areas of automated theorem proving.

Being able to disprove formulae is of key practical importance and various model building approaches and tools are available to disprove formulae by showing

R. A. Schmidt $(\otimes)$

School of Computer Science, The University of Manchester, Manchester, UK

e-mail: renate.schmidt@manchester.ac.uk,schmidt@cs.man.ac.uk

B. Pientka

School of Computer Science, McGill University, Montreal, Canada

e-mail: bpientka@cs.mcgill.ca 
the existence of finite models or finite model representations. The complementary problem of automatically determining the absence of finite models, that is, finite unsatisfiability, is the focus of the first paper. Koen Claessen and Ann Lillieström introduce an approach for tackling finite unsatisfiability based on exploiting known mathematical techniques and turning them into a fully automatic tool, called Infinox. The realisation involves non-trivial analysis of the given problem and clever search for entities which satisfy properties that imply the domain of any interpretation must be infinite (if one exists). To make the tool practically feasible various techniques and heuristics are developed to restrict the complexity and the search. The implementation involves calls to a finite model finder (namely Paradox) and a firstorder theorem prover (namely E). The results of extensive experiments on the TPTP problem library demonstrate finite unsatisfiability is a promising new direction of research in automated deduction.

The next paper integrates inductive theorem provers with term rewriting termination tools. Carsten Fuhs, Jürgen Giesl, Michael Parting, Peter Schneider-Kamp and Stephan Swiderski present a new technique in the dependency pair framework for automatically proving termination of term rewriting systems. The approach can handle systems where termination depends partially on inductive arguments. It has been implemented in the AProVE tool coupled with the inductive prover ACL2 and the own inductive prover of AProVE. The test results are very positive showing that 16 out of 19 previously unsolved term rewriting systems, extracted from the international corpus TPDB of termination problems, are now proved automatically.

SMT solvers provide efficient tools for integrated propositional reasoning and reasoning modulo domain-specific theories such as linear arithmetic and the theory of arrays, while provers based on resolution and superposition are strong at reasoning on first-order problems including equalities. Maria Paola Bonacina, Christopher A. Lynch and Leonardo de Moura develop a new DPLL(T)-style calculus where SMT solving is combined with generic inference systems based on superposition and resolution. Theories can be built-in theories, that are handled by special purpose theory reasoners, or axiomatised theories given by general clauses, that are handled by the generic first-order system. Additionally it is possible to perform speculative inferences based on specified conjectures. It is shown that the calculus is refutationally complete if background theory symbols only occur in ground clauses of the axiomatised theory, and the non-ground clauses are variable inactive. The approach provides decision procedures for a number of theories that axiomatise type systems in software verification for which speculative inferences are crucial.

The final paper is about the combination of superposition-based theorem proving and instance based methods. Peter Baumgartner and Uwe Waldmann present a calculus, called $\mathcal{M} \mathcal{E}+$ Sup, combining the ordered superposition calculus and the model evolution calculus for first-order logic with equality. As a first-order generalisation of the DPLL approach, the model evolution calculus is well-suited for model building, whereas the superposition calculus with sophisticated inference control mechanisms and a general notion of redundancy is well-suited for efficient equality reasoning. The $\mathcal{M} \mathcal{E}+$ Sup calculus combines these features into a powerful, integrated framework. It is shown to be both statically and dynamically sound and refutationally complete. 
We thank the authors of the submitted papers and the programme committee of CADE-22, and are especially gratefully to the expert reviewers who agreed to review the papers submitted to this special issue for their diligence and timely effort.

Manchester and Montreal, November 2010

\section{Reference}

1. Schmidt, R.A. (ed.): Automated Deduction-CADE-22, 22nd International Conference on Automated Deduction, Montreal, Canada, August 2-7, 2009. Proceedings. Lecture Notes in Artificial Intelligence, vol. 5663. Springer, New York (2009) 